\title{
Riding a spiral wave: Numerical simulation of spiral waves in a comoving frame of reference
}

\author{
A. J. Foulkes \\ Department of Computer Science, University of Liverpool, Ashton Building, Ashton Street, Liverpool L69 3BX, United Kingdom \\ V. N. Biktashev \\ Department of Mathematical Sciences, University of Liverpool, Mathematical Sciences Building, \\ Peach Street, Liverpool L69 7ZL, United Kingdom \\ (Received 25 January 2010; published 15 April 2010)
}

\begin{abstract}
We describe an approach to numerical simulation of spiral waves dynamics of large spatial extent, using small computational grids.

DOI: 10.1103/PhysRevE.81.046702

PACS number(s): 02.70. $-\mathrm{c}, 05.10 .-\mathrm{a}, 82.40 . \mathrm{Ck}, 82.40 . \mathrm{Bj}$
\end{abstract}

\section{INTRODUCTION}

Spiral waves are a type of self-organization observed in a large variety of spatially extended, thermodynamically nonequilibrium systems of physical, chemical and biological nature [1-19], where wave propagation is supported by a source of energy stored in the medium. If the system can be considered spatially uniform and isotropic and its properties do not depend on time, the corresponding mathematical models possess corresponding symmetries. For many practical applications, considerable interest is in nonstationary dynamics of spiral waves, which is usually defined separately either as drift, which is displacement of the average position of the core of the spiral with time due to external symmetrybreaking perturbations, or meandering, which is spontaneous symmetry breaking due to internal instability rather than external forces and which is manifested by complicated movement of the spiral with the average position of the core typically unmoved.

The numerical simulation of drift and meander of spiral waves, particularly when models are complicated and high accuracy is required, can be challenging. There are some theoretical considerations which suggest some way of dealing with this challenge. So it has been observed that as far as drift is concerned, spiral waves behave like particle-like objects, which results from effective localization of the critical eigenfunctions of the adjoint linearized operator [20-24], so it should be sufficient to do the computations only around the core of the spiral to predict its drift. On the other hand, in the absence of external symmetry-breaking perturbations, meandering of spirals can be understood by explicitly referring to the Euclidean symmetry of the unperturbed problem [25-30]. Specifically, an idea of dynamics in the space of symmetry group orbits [31], when applied to a reactiondiffusion system of equations and the Euclidean symmetry group, leads to a description which is formally equivalent to considering the solution in a moving frame of reference (FoR) such that the spiral wave maintains a certain position and orientation in this frame [29]. We shall call it comoving FoR for short.

The purpose of this paper is to present a computational approach based on these considerations. We calculate the dynamics of the spiral wave in a comoving FoR; as a result, the core of the spiral never approaches the boundaries of the computation box, which allows computations of drift and meandering of large spatial extent using small numerical grids. A simple software implementation of this approach, which is based on the popular spiral wave simulator "EZSPIRAL" [32,33], and which we called "EZRide," is provided on the authors' website [34].

Our approach can be compared to the approach proposed by Beyn and Thummler [35] and further developed by Hermann and Gottwald [36]. Their approach also exploits symmetry group orbits but is different in some essential details. We shall discuss the similarities and differences when we will have introduced our method.

The structure of the paper is as follows. In Sec. II we lay out mathematical basics of the approach and briefly compare it with [35]. In Sec. III we describe the numerical method itself. In Sec. IV we illustrate the work of the method by simple and quick examples. The potential for numerical accuracy is demonstrated in Sec. V. The subsequent three section are dedicated to examples of applications of the methods to problems where the conventional methods would be struggling: Sec. VI for the degenerate case of meandering which results in "spontaneous drift" of spirals; Sec. VII for the dynamics near to, and beyond, the parametric boundary at which the core radius of the spiral becomes infinite; and Sec. VIII for drift caused by a symmetry-breaking perturbation. We conclude with a brief discussion of the results in Sec. IX.

\section{SYMMETRY GROUP REDUCTION}

Following [29], we start from a perturbed reactiondiffusion system of equations in a plane,

$$
\frac{\partial \mathbf{u}}{\partial t}=\mathbf{D} \nabla^{2} \mathbf{u}+\mathbf{f}(\mathbf{u})+\mathbf{h}(\mathbf{u}, \nabla \mathbf{u}, \vec{r}, t),
$$

where $\mathbf{u}=\left(u^{(1)}, \ldots, u^{(n)}\right)^{\top}=\mathbf{u}(\vec{r}, t) \in \mathbb{R}^{n}$ is a column vector of reagent concentrations varying in space and time, $\mathbf{f}=\mathbf{f}(\mathbf{u})$ is a column vector of reaction rates, $\mathbf{D} \in \mathbb{R}^{n \times n}$ is the matrix of diffusion coefficients, $\mathbf{h} \in \mathbb{R}^{n}$ represents symmetry-breaking perturbations, $\|\mathbf{h}\| \ll 1, n \geq 2$, and $\vec{r}=(x, y) \in \mathbb{R}^{2}$. If $\mathbf{h}=\mathbf{0}$, then Eq. (1) is equivariant with respect to Euclidean transformations of the spatial coordinates $\vec{r}$.

The following technical discussion is necessary to place our method in the context of other works in the field. Readers not interested in technical details may skip down to system (16). 
The idea of the symmetry group reduction is convenient to describe if we view Eq. (1) as an ordinary differential equation $(\mathrm{ODE})$ in a suitably chosen functional space $\mathcal{B}$,

$$
\frac{d \mathbf{U}}{d t}=\mathbf{F}(\mathbf{U})+\mathbf{H}(\mathbf{U}, t)
$$

where $\mathbf{U}: \mathbb{R} \rightarrow \mathcal{B}$ represents the dynamic field $\mathbf{u}, \mathbf{F}: \mathcal{B} \rightarrow \mathcal{B}$ represents the unperturbed right-hand side $\mathbf{D} \nabla^{2} \mathbf{u}+\mathbf{f}$, and $\mathbf{H}: \mathcal{B} \times \mathrm{R} \rightarrow \mathcal{B}$ represents the perturbation $\mathbf{h}$.

Let us suppose that Eq. (2) at $\mathbf{h}=\mathbf{0}$ is equivariant with respect to a representation $\mathrm{T}$ of a Lie group $\mathcal{G}$ in $\mathcal{B}$. This means that for any $g \in \mathcal{G}$ and any $\mathbf{U} \in \mathcal{B}$, we have

$$
\mathbf{F}(\mathrm{T}(g) \mathbf{U})=\mathrm{T}(g) \mathbf{F}(\mathbf{U}) .
$$

In our case, $\mathcal{G}=\mathrm{SE}(2)$, the special Euclidean transformations of the plane $\mathbb{R}^{2} \rightarrow \mathbb{R}^{2}$ (including translations and rotations), and $\mathrm{T}$ is its representation in the space of functions $\mathbf{u}(\vec{r})$ defined on this plane, acting as

$$
\mathrm{T}(g) \mathbf{u}(\vec{r})=\mathbf{u}\left(g^{-1} \vec{r}\right) .
$$

We consider a subset $\mathcal{B}_{0} \subset \mathcal{B}$ such that $\mathcal{G}$ acts freely on $\mathcal{B}_{0}$; i.e., for a $\mathbf{U} \in \mathcal{B}_{0}$, any nontrivial transformation $g \in \mathcal{G}$ changes $\mathbf{U}$; and in other words, $\mathrm{T}(g) \mathbf{U}=\mathbf{U} \Rightarrow g=\mathrm{id}$. In the terminology of $[31], \mathcal{B}_{0}$ is the principal stratum of $\mathcal{B}$, corresponding to the trivial isotropy subgroups. In our case, this means that the graph of the function $\mathbf{u}(\vec{r}) \in \mathcal{B}_{0}$ is devoid of any rotational or translational symmetry, which is of course true for functions describing single-armed spiral waves.

It is straightforward that at $\mathbf{H}=\mathbf{0}$, the set $\mathcal{B}_{0}$ is an invariant set of Eq. (2). Moreover, we shall restrict our consideration to such perturbations $\mathbf{H}(t)$ that resulting solutions $\mathbf{U}(t)$ remain in $\mathcal{B}_{0}$ for all $t$. This means, that the perturbations are supposed to be so small that they cannot impose incidental symmetry on the otherwise unsymmetric spiral wave solutions.

A group orbit of a given $\mathbf{U}$ is defined as the set $\mathrm{T}(\mathcal{G}) \mathbf{U}$ $=\{\mathrm{T}(g) \mathbf{U} \mid g \in \mathcal{G}\}$. That is, it is a set of all such functions $\mathbf{u}(\vec{r})$ that can be obtained from one another by applying an appropriate Euclidean transformation to $\vec{r}$. A group orbit is a manifold in $\mathcal{B}_{0}$, of a dimensionality equal to $d=\operatorname{dim} \mathcal{G}$ less the dimensionality of the isotropy group. In our case, $\operatorname{dim} \operatorname{SE}(2)=3$, the isotropy group is trivial and the orbits are smooth three-dimensional manifolds.

From the definition of the set $\mathcal{B}_{0}$ it follows that this set is foliated by group orbits. The principal assumption for the following analysis is that there exists an open subset $\mathcal{S} \subset \mathcal{B}_{0}$, also invariant with respect to $\mathcal{G}$, in which the foliation has a global transversal section, i.e., we can select one representative from each orbit in $\mathcal{S}$, such that all such representatives form a smooth manifold $\mathcal{M} \subset \mathcal{S}$, which is everywhere transversal to the group orbits. We call this manifold a representative manifold (RM). That would mean that any orbit in $\mathcal{S}$ crosses $\mathcal{M}$ transversally and exactly once. Hence

$$
\forall \mathbf{U} \in \mathcal{S}, \quad \exists !(g, \mathbf{V}) \in \mathcal{G} \times \mathcal{M}: \quad \mathbf{U}=\mathrm{T}(g) \mathbf{V} .
$$

The RM has codimensionality equal to the dimensionality of the group orbits, i.e., in our case codim $\mathcal{M}=d=3$. It is assumed to be smooth and we expect that it can locally be described by equations $\mu_{\ell}(\mathbf{V})=0, \ell=1, \ldots d$, where functions $\mu_{\ell}: \mathcal{B} \rightarrow \mathbb{R}$, i.e., are functionals when interpreted in terms of original reaction-diffusion Eq. (1).

A convenient pictorial interpretation for our case is in terms of spiral wave solutions and their tips. Suppose the conditions $\mu_{1}(\mathbf{V})=0, \mu_{2}(\mathbf{V})=0$ determine that the tip of the spiral wave is located at the origin, and condition $\mu_{3}(\mathbf{V})=0$ fixes its orientation, so $\mathcal{M}$ consists of such functions $\mathbf{V}$ that look like spiral waves which have the tip exactly at the origin and in a standard orientation. Then Eq. (5) states that any spiral wave solution $\mathbf{u}(\vec{r})$, considered at a fixed moment of time, can be transformed by a Euclidean transformation, in a unique way, to a solution $\mathbf{v}(\vec{r})$ which has its tip at the origin and in the standard orientation. This is equivalent to saying that $\mathbf{v}(\vec{r})$ is the same as $\mathbf{u}(\vec{r})$ only considered in a different system of coordinates, with the origin at the tip of $\mathbf{u}(\vec{r})$ and oriented accordingly to the orientation of that tip. We shall say this is the system of coordinates attached to the tip. An example of $\mu_{\ell}$, as used, e.g., in [29], is

$$
\begin{gathered}
\mu_{1}[\mathbf{v}(\vec{r})]=v^{\left(l_{1}\right)}(\overrightarrow{0})-u_{*}, \\
\mu_{2}[\mathbf{v}(\vec{r})]=v^{\left(l_{2}\right)}(\overrightarrow{0})-v_{*}, \\
\mu_{3}[\mathbf{v}(\vec{r})]=\partial_{x} v^{\left(l_{3}\right)}(\overrightarrow{0}),
\end{gathered}
$$

where $\left\{l_{1}, l_{2}, l_{3}\right\} \subset\{1, \ldots, n\}$ are suitably chosen components, and $l_{1} \neq l_{2}$. This means that the tip of $\mathbf{u}(\vec{r})$ is defined as the point of intersection of isolines of the components $l_{1}$ and $l_{2}$ of the field $\mathbf{u}$ at appropriately chosen levels $u_{*}$ and $v_{*}$ respectively, and the orientation of the attached coordinate system is such that gradient of component $l_{3}$ (which may or may not coincide with $l_{1}$ or $l_{2}$ ) is along the $y$-axis in that system. This choice of $\mu_{\ell}$ is of course not prescriptive, and later in this paper we shall consider some variations.

Regardless of the exact definition of the tip, i.e., choice of functionals $\mu_{\ell}$, an essential assumption that we have to make is that our spiral waves have one tip only, otherwise there would be more than one way to transform them to the standard position or to chose the attached system of coordinates. Hence the reason for a further constraint to the subset $\mathcal{S} \subset \mathcal{B}_{0}$, which we now can define as consisting of such onetip spiral wave solutions, or functions that look like it: without such constraint, the whole set $\mathcal{B}_{0}$ includes solutions with no tips or more than one tip, for which decomposition (5) would not hold. As before, we assume that set $\mathcal{S}$ is invariant with respect to dynamic Eq. (2) for not too big $\|\mathbf{H}\|$; that is, if $\mathbf{U}(0) \in \mathcal{S}$, then $\mathbf{U}(t) \in \mathcal{S}$ for all $t>0$ and $\|\mathbf{H}\|<H_{\max }$.

A further restriction is on the manifold $\mathcal{M}$. It is easy to see that equations such as Eq. (6) may not be sufficient to define the manifold with the required property that any orbit crosses it only once. For instance, if $\mathbf{v}(\vec{r})$ satisfies Eq. (6), then $\mathbf{v}(-\vec{r})$ also satisfies it, so a rotation by $180^{\circ}$ around the origin transfers a point on $\mathcal{S}$ to another point on $\mathcal{S}$. So to make representation (5) unique, rather than just requiring that the gradient of the $l_{3}$ component of $\mathbf{v}(\vec{r})$ is along the $y$ axis, one would need to specify in which direction it is, say add to the definition of $\mathcal{M}$ by the equations $\mu_{1,2,3}[\mathbf{v}]=0$ a further inequality 


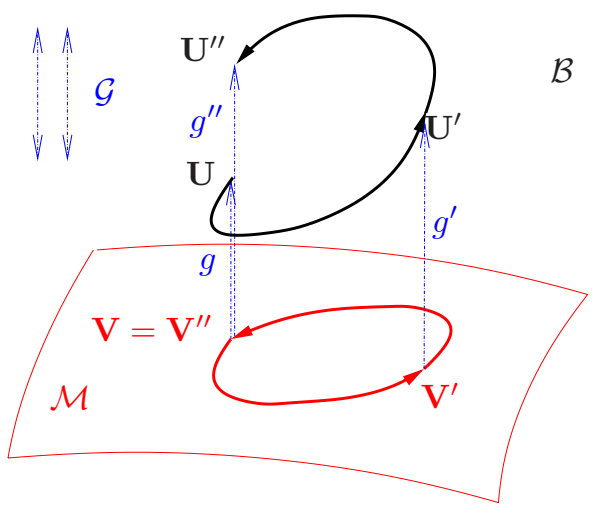

FIG. 1. (Color online) Sketch of skew-product decomposition of an equivariant flow using a representative manifold $\mathcal{M}$, which has exactly one transversal intersection with every group orbit $g \in \mathcal{G}$ within the relevant stratum of the phase space $\mathcal{B}$ and is diffeomorphic to the orbit manifold. Trajectory $\left(\mathbf{U}, \mathbf{U}^{\prime}, \mathbf{U}^{\prime \prime}\right)$ of an equivariant flow in $\mathcal{B}$ is a relative periodic orbit, since it projects onto the trajectory $\left(\mathbf{V}, \mathbf{V}^{\prime}, \mathbf{V}^{\prime \prime}=\mathbf{V}\right)$ on $\mathcal{M}$ which is periodic. The flow on $\mathcal{M}$ is devoid of symmetry $\mathcal{G}$.

$$
\mu_{4}[\mathbf{v}]>0, \quad \text { where } \mu_{4}[\mathbf{v}(\vec{r})]=\partial_{y} v^{\left(l_{3}\right)} .
$$

This comment extends to the variations of Eq. (6) which we consider later.

By performing decomposition (5) for every $t \geq 0$, we decompose motion in $\mathcal{S}$ to motion along the RM and motion along group orbits which are transversal to the RM (see illustration in Fig. 1).

So for all $t \geq 0$, we have

$$
\mathbf{U}(t)=\mathrm{T}(g) \mathbf{V}(t)
$$

Substituting Eq. (7) into Eq. (2) and applying $\mathrm{T}\left(g^{-1}\right)$ to both sides, we get

$$
\mathrm{T}\left(g^{-1}\right) \frac{d \mathrm{~T}(g)}{d t} \mathbf{V}+\frac{\partial \mathbf{V}}{\partial t}=\mathbf{F}(\mathbf{V})+\tilde{\mathbf{H}}(\mathbf{V}, g, t)
$$

where

$$
\tilde{\mathbf{H}}(\mathbf{V}, g, t)=\mathrm{T}\left(g^{-1}\right) \mathbf{H}(\mathrm{T}(g) \mathbf{V}, t) .
$$

We note that if $\mathbf{H = 0}$, the right-hand side of Eq. (8) is independent of $g$.

By the assumptions made, intersection of the group orbit $\mathrm{T}(\mathcal{G}) \mathbf{V}$ with the manifold $\mathcal{M}$ at the point $\mathbf{V}$ is transversal. This means that the vectors $\mathbf{F}(\mathbf{V})$ and $\tilde{\mathbf{H}}(\mathbf{V}, g, t)$ can be uniquely decomposed into the sums of the components along the group and along the manifold,

$$
\begin{gathered}
\mathbf{F}(\mathbf{V})=\mathbf{F}_{\mathcal{G}}(\mathbf{V})+\mathbf{F}_{\mathcal{M}}(\mathbf{V}), \\
\widetilde{\mathbf{H}}(\mathbf{V}, g, t)=\mathbf{H}_{\mathcal{G}}(\mathbf{V}, t)+\mathbf{H}_{\mathcal{M}}(\mathbf{V}, t) .
\end{gathered}
$$

Hence Eq. (8) splits into two components, along the RM and along the group orbit (GO),

$$
(\mathrm{RM}) \quad \frac{\partial \mathbf{V}}{\partial t}=\mathbf{F}_{\mathcal{M}}(\mathbf{V})+\mathbf{H}_{\mathcal{M}}(\mathbf{V}, t),
$$

$$
\text { (GO) } \mathrm{T}\left(g^{-1}\right) \frac{d \mathrm{~T}(g)}{d t} \mathbf{V}=\mathbf{F}_{\mathcal{G}}(\mathbf{V})+\mathbf{H}_{\mathcal{G}}(\mathbf{V}, t) \text {. }
$$

Note that Eq. (11a) is the equation on the infinitedimensional manifold $\mathcal{M}$, i.e., corresponds to a partial differential equation (PDE), whereas the left- and right-hand sides of Eq. (11b) are in the tangent space to the finitedimensional group orbits, and the dynamic variable $g$ is an element of the finite-dimensional manifold $\mathcal{G}$, so Eq. (11b) is in fact a system of ordinary differential equations of order $d=\operatorname{dim} \mathcal{G}$.

At this point we comment on what we see as a significant difference between our approach and that proposed by Beyn and Thummler (BT) [35]. Using our notation, in place of our "pinning" conditions $\mu_{\ell}(\mathbf{V})=0, \ell=1, \ldots, d$, they defined "phase conditions" of the form $\mu_{\ell}(\mathbf{V}, g)=0$ (see Eq. (2.22) in [35]), subsequently further generalized to $\mu_{\ell}(\mathbf{V}, g, d g / d t)$ $=0$ (Eq. (2.33) in [35]). This means that their decomposition $\mathbf{U}=\mathrm{T}(g) \mathbf{V}$ is not uniquely determined by the current state $\mathbf{U}$ but depends on history. Such generalization may have its advantages and, apparently, works well for relative equilibria, i.e., steadily rotating spirals $[35,36]$. However, the situation is different if the solution is a meandering spiral, i.e., is periodic with period $P$ in the orbit space (as illustrated in Fig. 1). This means that $\mathbf{U}(t+P)$ is equivalent to $\mathbf{U}(t)$ up to some Euclidean transformation. In our approach, it is then guaranteed, that $\mathbf{V}(t+P)=\mathbf{V}(t)$, as by Eq. (5), $\mu_{\ell}\left(\mathrm{T}\left(g^{-1}\right) \mathbf{U}\right)=0$ has a unique solution for $g$ at a given $\mathbf{U}$. However, in the BT approach, typically $\mathbf{V}(t+P) \neq \mathbf{V}(t)$, since $\mu_{\ell}\left(\mathrm{T}\left(g^{-1}\right) \mathbf{U}, g, d g / d t\right)=0$ does not uniquely define $g$, as $d g / d t$ is not fixed. So in our approach, study of meandering spirals reduces to study of periodic solutions for $\mathbf{V}(t)$, but it does not do so in the BT approach.

A practical approach to the problem of decomposing the vector fields as in Eq. (10) is as follows. Equation (11) together with the definition of the RM via functionals $\mu_{\ell}$ can be rewritten in an equivalent form

$$
\frac{\partial \mathbf{V}}{\partial t}=\mathbf{F}+\tilde{\mathbf{H}}+\mathbf{A}
$$

$$
\begin{gathered}
\mu_{\ell}(\mathbf{V}(t))=0, \quad \ell=1, \ldots, d, \\
\mathrm{~T}\left(g^{-1}\right) \frac{d \mathrm{~T}(g)}{d t} \mathbf{V}=-\mathbf{A},
\end{gathered}
$$

where $\mathbf{A}=\mathbf{A}(\mathbf{V}, t)=-\mathbf{F}_{\mathcal{G}}(\mathbf{V})-\mathbf{H}_{\mathcal{G}}(\mathbf{V}, t)$ is a vector belonging to the three-dimensional tangent space of the group orbit $\mathrm{T}(\mathcal{G})(\mathbf{V})$ at $\mathbf{V}$. In this formulation, at any given moment of time, Eqs. (12a) and (12b) together define the evolution of $\mathbf{V}$ and the current value of the vector $\mathbf{A}$, whereas Eq. (12c) defines the evolution of $g$.

By definition, vector $\mathbf{A}$ is a result of action of a linear combination of the generators of the Lie group $\mathrm{T}(\mathcal{G})$ as linear operators on $\mathbf{V}$. To write the explicit expression for the general form $\mathbf{A}$ for our case, let us introduce coordinates $(\vec{R}, \Theta)$ 
on $\mathcal{G}=\mathrm{SE}(2)$, where $\vec{R}=(X, Y)$ is the translation vector, $\Theta$ is the rotation angle and a group element acts as

$$
g=(\vec{R}, \Theta): \vec{r} \mapsto \vec{R}+e^{\hat{\gamma}^{\Theta}} \vec{r},
$$

where $\hat{\gamma}=\left[\begin{array}{cc}0 & -1 \\ 1 & 0\end{array}\right]$, so $\exp (\hat{\gamma} \Theta)$ is the matrix of rotation by angle $\Theta$.

Using this representation, differentiating the definition of $\mathrm{T}(g) \mathbf{v}$ given by Eq. (4), and substituting the result into Eq. (12c), we get

$$
\mathbf{A}=\omega \partial_{\theta} \mathbf{v}+(\vec{c} \cdot \nabla) \mathbf{v}
$$

where

$$
\omega=\dot{\Theta}, \quad \vec{c}=e^{-\hat{\gamma} \Theta} \dot{\vec{R}},
$$

and $\theta$ is the polar angle in the $(x, y)$ plane, so $\partial_{\theta}=x \partial_{y}-y \partial_{x}$.

With this result, system (12) in the original PDE notation states that

$$
\begin{aligned}
& \frac{\partial \mathbf{v}}{\partial t}=\mathbf{D} \nabla^{2} \mathbf{v}+\mathbf{f}(\mathbf{v})+\mathbf{h}\left(\mathbf{v}, e^{\hat{\gamma} \Theta} \nabla \mathbf{v}, \vec{R}+e^{\hat{\gamma}^{\Theta}} \vec{r}, t\right) \\
& +(\vec{c} \cdot \nabla) \mathbf{v}+\omega \frac{\partial \mathbf{v}}{\partial \theta}, \\
& v^{\left(l_{1}\right)}(\overrightarrow{0}, t)=u_{*}, \quad v^{\left(l_{2}\right)}(\overrightarrow{0}, t)=v_{*}, \\
& \frac{\partial v^{\left(l_{3}\right)}(\overrightarrow{0}, t)}{\partial x}=0, \quad \frac{\partial v^{\left(l_{3}\right)}(\overrightarrow{0}, t)}{\partial y}>0, \\
& \frac{d \Theta}{d t}=\omega, \quad \frac{d \vec{R}}{d t}=e^{\hat{\gamma} \Theta} \vec{c},
\end{aligned}
$$

where the dynamic variables are $\mathbf{v}(\vec{r}, t), \vec{c}(t), \omega(t), \vec{R}(t)$, and $\Theta(t)$.

In terms of the tip of the wave, Eq. (16a) is the original reaction-diffusion Eq. (1) written in the comoving FoR, Eqs. (16b) and (16c) define the attachment (pinning) of the tip to this FoR, and Eqs. (16d) describe the movement of the FoR and, therefore, of the tip.

Equations (16b) and (16c) imply that the position $\left(x_{\text {tip }}, y_{\text {tip }}\right)$ and orientation $\Phi$ of the tip during calculations in the laboratory FoR are defined as

$$
\begin{gathered}
u^{\left(l_{1}\right)}\left(x_{\text {tip }}(t), y_{\text {tip }}(t), t\right)=u_{*}, \\
u^{\left(l_{2}\right)}\left(x_{\text {tip }}(t), y_{\text {tip }}(t), t\right)=v_{*}, \\
\Phi(t)=\arg \left(\left(\partial_{x}+i \partial_{y}\right) u^{\left(l_{3}\right)}\left(x_{\text {tip }}(t), y_{\text {tip }}(t), t\right)\right)
\end{gathered}
$$

and the comoving FoR is chosen so that in it, $\left(x_{\text {tip }}, y_{\text {tip }}\right)$ $=(0,0)$ and $\Phi=\pi / 2$ at all times. Unlike other equations of system [Eq. (16)], these are not prescriptive and is essentially an arbitrary choice, dictated by properties of particular systems. We shall refer to pinning conditions (16b) and (16c) as "choice 1," as below we shall consider a variation in these, which we call "choice 2 ."
When $\mathbf{h}=\mathbf{0}$, system (16) decouples, as its upper part including Eqs. (16a)-(16c) becomes independent of the lower part [Eq. (16d)]. This is the "skew-product" decomposition, the upper part describing the dynamics in the space of group orbits, so-called "quotient system," and the lower part the "symmetry group extension," i.e., dynamics along the group, which depends on but does not affect the quotient dynamic. The connection between the quotient system and the group extension is via the dynamic variables $(\vec{c}, \omega)$; in the following, we refer to these three quantities as "quotient data" for brevity.

The skew-product representation has been useful for the analysis of various types of meander of spiral waves $[29,30,37,38]$. Note that the approach used in [30,38] (also see references therein) is based on the assumption that the meandering pattern in question is considered in the vicinity of a bifurcation from the rigidly rotating spiral wave solution so that the quotient dynamics can be reduced to the center manifold, hence instead of Eqs. (16a)-(16c), these studies considered normal forms on the corresponding center manifolds. However, as noted in [39], the centre manfold theorem is not applicable for spiral waves, so this approach seems to be fundamentally flawed. This technical difficulty of course does not in any way affect the validity of system (16), which, as we have just demonstrated, is derived by elementary means without recourse to any bifurcations.

In the rest of the paper, we consider system (16) as a computational tool rather than an instrument of theoretical analysis. The disadvantage of original system (1) as a computational tool is that it requires a big computational grid to simulate dynamics of a spiral in an infinite medium, particularly when the tip of the spiral performs excursions to large distances. This is actually not necessary, as the dynamics of the spiral is mostly determined by the events in some finite vicinity of its tip [24]. System (16) takes advantage of this property so that the PDE calculations are done always in some fixed vicinity of the spiral wave, whereas the movement of the tip is described by the ODE part.

\section{NUMERICAL IMPLEMENTATION}

\section{A. Discretization}

We use time discretization with constant step $\Delta_{t}$ and square spatial grid with step $\Delta_{x}$, covering spatial domain $(x, y) \in[-L / 2, L / 2]^{2}$, so that

$$
\begin{gathered}
\mathbf{v}\left(\left(i-i_{0}\right) \Delta_{x},\left(j-j_{0}\right) \Delta_{x}, k \Delta_{t}\right) \sim \hat{\mathbf{v}}_{i, j}^{k}=\left(\hat{v}_{i, j}^{(l), k} \mid l=1, \ldots, n\right), \\
i=0, \ldots, N_{x}, \quad j=0, \ldots, N_{y}, \\
N_{x}=N_{y}=L / \Delta_{x},
\end{gathered}
$$

and the grid coordinates of the origin are

$$
i_{0}=\left(N_{x}+1\right) / 2, \quad j_{0}=\left(N_{y}+1\right) / 2
$$

(we only use odd values of $N_{x}=N_{y}$ ). We designate the $k$ th time layer of the numerical solution as $\hat{\mathbf{V}}^{k}$ $=\left(\hat{\mathbf{v}}_{i, j}^{k} \mid i=1, \ldots, N_{x}, j=1, \ldots, N_{y}\right)$. We discretize the ODE dynamic variables on the same time grid, i.e., $\vec{R}\left(k \Delta_{t}\right) \sim \hat{\vec{R}}^{k}$, etc. 


\section{B. Operator splitting}

We rewrite Eq. (16a) in the form

$$
\frac{\partial \mathbf{v}}{\partial t}=\mathcal{F}[\mathbf{v}]+\mathcal{H}[\mathbf{v} ; \vec{R}, \Theta]+\mathcal{A}[\mathbf{v} ; \vec{c}, \omega]
$$

where differential operators $\mathcal{F}, \mathcal{H}$, and $\mathcal{A}$ are defined as

$$
\begin{gathered}
\mathcal{F}[\mathbf{v}]=\mathbf{D} \nabla^{2} \mathbf{v}+\mathbf{f}(\mathbf{v}), \\
\mathcal{H}[\mathbf{v} ; \vec{R}, \Theta]=\mathbf{h}\left(\mathbf{v}, e^{\hat{\gamma}^{\Theta}} \cdot \nabla \mathbf{v}, \vec{R}+e^{\hat{\gamma} \Theta} \vec{r}, t\right), \\
\mathcal{A}[\mathbf{v} ; \vec{c}, \omega]=(\vec{c} \cdot \nabla) \mathbf{v}+\omega \frac{\partial \mathbf{v}}{\partial \theta}=\left(c_{x}-\omega y\right) \frac{\partial \mathbf{v}}{\partial x}+\left(c_{y}+\omega x\right) \frac{\partial \mathbf{v}}{\partial y} .
\end{gathered}
$$

Let $\hat{\mathcal{F}}, \hat{\mathcal{H}}$, and $\hat{\mathcal{A}}$ be discretizations of $\mathcal{F}, \mathcal{H}$, and $\mathcal{A}$. Our computations proceed as follows:

$$
\begin{gathered}
\hat{\mathbf{V}}^{k+1 / 3}=\hat{\mathbf{V}}^{k}+\Delta_{t} \hat{\mathcal{F}}\left(\hat{\mathbf{V}}^{k}\right), \\
\hat{\mathbf{V}}^{k+2 / 3}=\hat{\mathbf{V}}^{k+1 / 3}+\Delta_{t} \hat{\mathcal{H}}\left(\hat{\mathbf{V}}^{k+1 / 3}, \hat{\vec{R}}^{k}, \hat{\Theta}^{k}\right), \\
\hat{\mathbf{V}}^{k+1}=\hat{\mathbf{V}}^{k+2 / 3}+\Delta_{t} \hat{\mathcal{A}}\left(\hat{\mathbf{V}}^{k+2 / 3}, \hat{\vec{c}}^{k+1}, \hat{\omega}^{k+1}\right), \\
\mu_{1,2,3}\left(\hat{\mathbf{V}}^{k+1}\right)=0, \quad \mu_{4}\left(\hat{\mathbf{V}}^{k+1}\right)>0, \\
\hat{\Theta}^{k+1}=\hat{\Theta}^{k}+\Delta_{t} \omega^{k+1}, \\
\hat{\vec{R}}^{k+1}=\hat{\vec{R}}^{k}+\Delta_{t} e^{\hat{\boldsymbol{\gamma}} \hat{\Theta}^{k+1}} \vec{c}^{k+1} .
\end{gathered}
$$

\section{Kinetics}

As specific examples, we consider two models, the FitzHugh-Nagumo (FHN) model [40,41],

$$
\mathbf{f}:\left[\begin{array}{l}
u \\
v
\end{array}\right] \mapsto\left[\begin{array}{c}
\alpha^{-1}\left(u-u^{3} / 3-v\right) \\
\alpha(u+\beta-\gamma v)
\end{array}\right]
$$

and Barkley's model [32,42],

$$
\mathbf{f}:\left[\begin{array}{l}
u \\
v
\end{array}\right] \mapsto\left\{\begin{array}{c}
c^{-1} u(1-u)[u-(v+b) / a] \\
u-v
\end{array}\right\},
$$

both with $\mathbf{D}=\left[\begin{array}{ll}1 & 0 \\ 0 & 0\end{array}\right]$.

\section{Reaction-diffusion step}

The computational scheme is designed as an extension to the standard approach to simulation of spiral waves. Specifically, we chose Barkley's EZ-SPIRAL [32,33,42] as the starting point and extended it to add the other computational steps. So reaction-diffusion step (20a) is as implemented in EZ-SPIRAL, with central five-point difference approximation of the Laplacian, without any features specific to the Barkley model, such as implicit treatment of the kinetic terms, and with appropriate modifications when FitzHugh-Nagumo model is used.

\section{E. Perturbations}

We consider one particular type of nonzero perturbation, the electrophoresis,

$$
\begin{gathered}
\mathbf{h}=\mathbf{E} \partial_{x} \mathbf{u}, \\
\tilde{\mathbf{h}}=\mathbf{E}\left[\cos (\Theta) \partial_{x} \mathbf{v}(r)-\sin (\Theta) \partial_{y} \mathbf{v}(r)\right],
\end{gathered}
$$

where $\mathbf{E}$ is a diagonal matrix, $\mathbf{E}=\left[\begin{array}{cc}E_{1} & 0 \\ 0 & E_{2}\end{array}\right],\|\mathbf{E}\| \ll 1$. For a reaction-diffusion system this perturbation can describe movement of the reagents in response to electric field with velocities $-E_{1}$ and $-E_{2}$ along the $x$ axis. For $\mathbf{E}=\epsilon \mathbf{D}$, this perturbation can also approximately describe the movement of an axially symmetric scroll ring. For a cylindrical system of coordinates $(r, \theta, z): x=r \cos \theta, y=r \sin \theta, z=z$, the diffusion term has the form $\mathbf{D} \nabla^{2} \mathbf{u}=\mathbf{D}\left(\partial_{r}^{2}+r^{-1} \partial_{r}+r^{-2} \partial_{\theta}^{2}+\partial_{z}^{2}\right) \mathbf{u}$, which for $\partial_{\theta}=0$ and large $r$ is equivalent to an unperturbed diffusion term with a two-dimensional Laplacian in $(r, z)$ plane plus a small perturbation $\frac{1}{r} \mathbf{D} \partial_{r} \mathbf{u}$. If the filament of the scroll is located at large values of $r \approx 1 / \epsilon$ and as the dynamics of the scroll is mostly determined by the events near its filament, then $1 / r$ can be approximately replaced with $\epsilon$.

Perturbation (23) violates only rotational symmetry of the problem, preserving symmetry with respect to translations in space and time. Hence $\tilde{\mathbf{h}}$ explicitly depends only on $\Theta$. This limitation is not principal and translation symmetrybreaking perturbations can be considered similarly, in which case $\tilde{\mathbf{h}}$ would also explicitly depend on $X, Y$ and/or $t$. We discretize the first spatial derivatives in the perturbation term using upwind second-order accurate differences, and use explicit Euler time stepping. In the absence of perturbations, $\mathbf{h}=\mathbf{0}$, perturbation step (20b) is of course omitted and $\hat{\mathbf{V}}^{k+2 / 3}=\hat{\mathbf{V}}^{k+1 / 3}$.

\section{F. Tip definition and pinning conditions}

Discretization of pinning conditions (16b) and (16c), using $l_{1}=l_{3}$, and the right-side first-order discretization of the $x$-derivative, gives

$$
\begin{gathered}
\hat{v}_{i_{0}, j_{0}}^{\left(l_{1}\right), k}=u_{*}, \\
\hat{v}_{i_{0}, j_{0}}^{\left(l_{2}\right), k}=v_{*}, \\
\hat{v}_{i_{0}+1, j_{0}}^{\left(l_{1}\right), k}=u_{*},
\end{gathered}
$$

where $\left(i_{0}, j_{0}\right)$ are grid coordinates of the origin. This works in principle but gives rather inaccurate and noisy approximations for $\omega$, which get worse for finer discretizations. This is typical for numerical differentiation. We overcome this by enhancing the spatial discretization step, by replacing condition $(24 c)$ with

$$
\hat{v}_{i_{1}, j_{1}}^{\left(l_{1}\right), k}=u_{*},
$$

where the grid point $\left(i_{1}, j_{1}\right)$ was chosen some way away from the center point, $\left(i_{1}, j_{1}\right)=\left(l_{0}, j_{0}\right)+\left(i_{\text {inc }}, j_{\text {inc }}\right)$. This means replacing third pinning condition (16c) with 


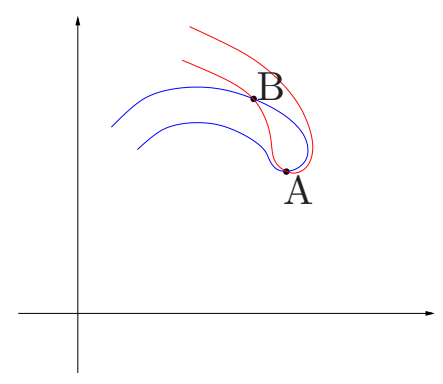

FIG. 2. (Color online) Nonuniqueness of the revised tip pinning condition.

$$
v^{\left(l_{1}\right)}\left(\vec{r}_{\text {inc }}, t\right)=u_{*},
$$

where $\vec{r}_{\text {inc }}=\left(\Delta_{x} i_{\text {inc }}, \Delta_{x} j_{\text {inc }}\right)$. Empirically, we have found that the length of the displacement $\left|\vec{r}_{\text {inc }}\right|$ should be of the order of, but not exceeding, one full wavelength of the spiral.

This revised orientation-pinning condition still does not define the position uniquely, as illustrated by Fig. 2. An extra inequality is required to distinguish between different solutions satisfying conditions (24a), (24b), and (25). We use

$$
\hat{v}_{i_{1}, j_{1}}^{\left(l_{1}\right), k}<v_{*} .
$$

corresponding to

$$
v^{\left(l_{1}\right), k}\left(\vec{r}_{\mathrm{inc}}, t\right)<v_{*} .
$$

Specifically, we chose $l_{1}=l_{3}=1$ and $l_{2}=2$. Conditions (27) and (28) then mean that the third pinning condition [Eqs. (25) and (26)] ensures that the front, rather than the back, of the excitation wave passes though the grid point $\left(i_{1}, j_{1}\right)$. So Eqs. (16b), (26), and (28), with discretizations (24) and (27) are our "choice 2 " pinning conditions.

The choice 1 and choice 2 pinning conditions define different RMs and different quotient data $\vec{c}(t), \omega(t)$, for the same solution $\mathbf{u}(\vec{r}, t)$. However, the two FoRs they define have a common origin and differ only by the orientation angle. So if $(\vec{c}, \omega)$ are quotient data for choice 1 pinning conditions, and $\left(\vec{c}^{\prime}, \omega^{\prime}\right)$ are quotient data for choice 2 pinning conditions, then we have

$$
\vec{c}=e^{\hat{\gamma}(\Phi-\pi / 2)} \vec{c}^{\prime}, \quad \omega=\omega^{\prime}+d \Phi / d t,
$$

where $\Phi$ the tip orientation angle in choice 2 comoving FoR, so $\Phi-\pi / 2$ is angle of one FoR against the other.

\section{G. Advection}

We use an upwind second-order accurate approximation of the spatial derivatives in $\hat{\mathcal{A}}$. Steps (20c) and (20d) are done in conjunction with each other. The discretization of $\hat{\mathbf{V}}^{k+1}$ at the tip pinning points, resulting from Eq. (20c), is used in the three equations [Eq. (20d)] to find the three unknowns $\hat{\vec{c}}_{x}^{k+1}, \hat{\vec{c}}_{y}^{k+1}$, and $\hat{\omega}^{k+1}$ so that the pinning conditions [Eq. (20d)] are always satisfied exactly (to the processor precision) after every step [52].

\section{H. Boundary conditions}

Since the boundaries in the comoving FoR do not represent any physical reality but are only a necessity of numeri- cal approximation, the results can only be considered to be reliable if they do not depend on the boundary conditions. So we use both Dirichlet and Neumann boundary conditions and compare the results. For Dirichlet conditions, we use boundary values of the resting state $\mathbf{v}_{r}$, such that $\mathbf{f}\left(\mathbf{v}_{r}\right)=\mathbf{0}$.

\section{Tip trajectory reconstruction}

Steps (20e) and (20f) are simple first-order implementations of the corresponding ODEs. The resulting $\hat{\Theta}$ is used in calculations of the $\mathcal{H}$ step when the perturbation is on. Otherwise, $\hat{\Theta}$ and $\hat{\vec{R}}$ are calculated only for the record.

\section{J. Some details of software implementation}

For stability purposes, we ensure that the following inequalities are observed during computations:

$$
\begin{gathered}
\left|c_{x}\right| \leq \frac{\Delta_{x}^{2}}{2 \Delta_{t}} \\
\left|c_{y}\right| \leq \frac{\Delta_{x}^{2}}{2 \Delta_{t}} \\
|\omega| \leq \frac{1}{N_{X} \Delta_{t}} .
\end{gathered}
$$

This is an empirical choice motivated by von Neumann stability analysis.

When the absolute values of $c_{x}$ and $c_{y}$ found in Eqs. (20c) and (20d) are beyond these limits then they are restricted to the intervals stated above. Also, we eliminated the need to restrict the values of $c_{x}$ and $c_{y}$ to their stability limits by moving the spiral wave solution so that the tip of the spiral wave is in the center of the box, using the standard EZSPIRAL's "mover" function, which performs translation of the solution by an integer number of grid steps, suitably extrapolating the solution where necessary near the boundaries.

For $\omega$, we implemented the restriction that if $|\omega|$ exceeded its maximum stability value, then we set $\omega=0$. Effectively this means that unless the orientation of the spiral wave is already very near the standard orientation satisfying Eq. (25) and inequality (27), the code computes a solution of the problem

$$
\begin{aligned}
& \frac{\partial \mathbf{v}}{\partial t}=\mathbf{D} \nabla^{2} \mathbf{v}+\mathbf{f}(\mathbf{v})+\mathbf{h}\left(\mathbf{v}, \nabla \mathbf{v}, \vec{R}+e^{\hat{\gamma} \Theta} \vec{r}, t\right)+(\vec{c} \cdot \nabla) \mathbf{v}, \\
& v^{\left(l_{1}\right)}(\overrightarrow{0}, t)=u_{*}, \quad v^{\left(l_{2}\right)}(\overrightarrow{0}, t)=v_{*}, \\
& \frac{d \vec{R}}{d t}=e^{\hat{\gamma}^{\Theta}} \vec{c}
\end{aligned}
$$

instead of Eq. (16). That is, it performs reduction by the subgroup of translations of the Euclidean group.

A typical run of the program in the interactive mode starts from obtaining a spiral wave solution in the standard "rideoff" mode, by solving initial-value problem (1). When the 

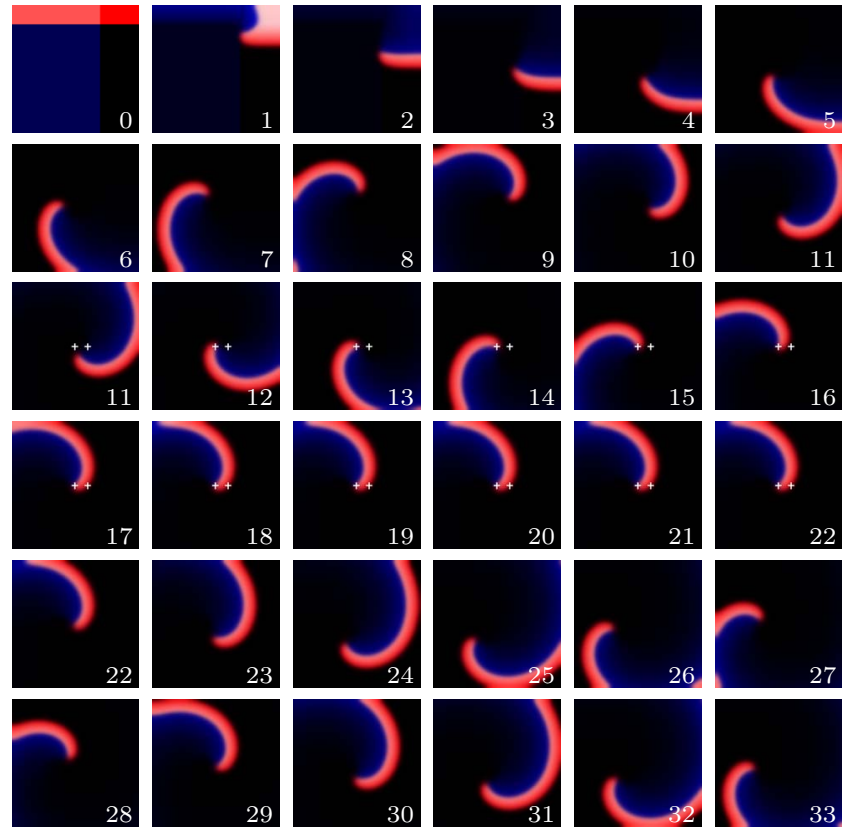

FIG. 3. (Color online) Three consecutive runs of Barkley model, $a=0.52, b=0.05, c=0.02, L=20, \Delta_{x}=1 / 5, \Delta_{t}=1 / 2000$, and $\vec{r}_{\text {inc }}$ $=(2,0)$. The runs $t \in[0,11]$ and $t \in[22,33]$ are direct simulations. The run $t \in[11,22]$ is a quotient system simulation, the pinning points are indicated by small white crosses. The third pinning condition is engaged at $t \approx 16.5$.

spiral wave is initiated so there is one tip in the solution, the user switches the program to the "ride-on" mode, with calculations according to the above scheme. On the switch, the program first of all moves the tip of the spiral to the center of the box via EZ-SPIRAL's "mover" function, i.e., by parallel translations of the solution, supplementing the missing pieces near boundaries by duplicating the existing boundary values. From then on, the spiral continues to rotate with its tip fixed at the center of the box, thus solving problem (30). In this regime, only the first two pinning conditions are satisfied, and only $c_{x}$ and $c_{y}$ are calculated and used, where as $\omega$ is calculated but replaced with zero, until it falls within the stability limit and the fourth inequality-type pinning condition is satisfied. From that point, the program proceeds in the fully engaged mode, calculating problem (16).

\section{PRIMARY EXAMPLES: RIGIDLY ROTATION AND MEANDER}

First we illustrate how our approach works using two examples. One example uses Barkley model with rigidly rotating spiral waves, and the other is FitzHugh-Nagumo model with meandering spiral waves.

Figure 3 illustrates the work of EZRide in the case of a rigidly rotating spiral wave. The panels represent three consecutive runs, in different regimes: the "direct numerical simulations" (DNSs) of system (1), then the "skew-product" calculation in the comoving FoR, and then again the DNS in the laboratory FoR. The skew-product calculation in turn consists of two parts. The first part is described by Eq. (30)

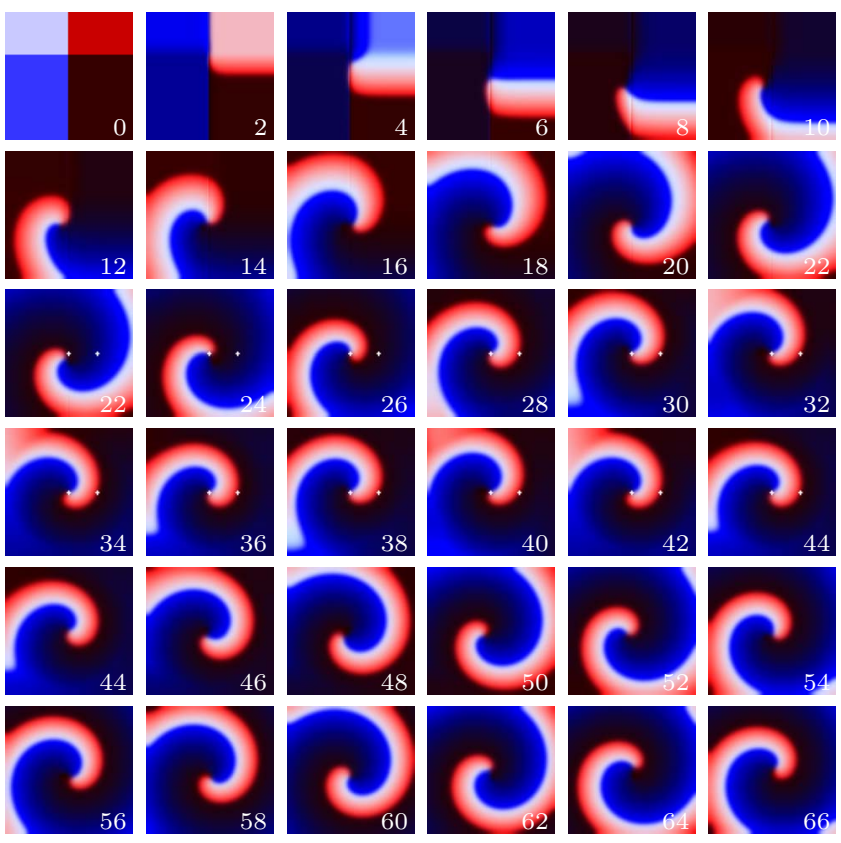

FIG. 4. (Color online) Three consecutive runs of FHN model, $\alpha=0.2, \quad \beta=0.7, \quad \gamma=0.5, \quad L=30, \quad \Delta_{x}=1 / 3, \quad \Delta_{t}=1 / 720, \quad$ and $\vec{r}_{\text {inc }}$ $=(20 / 3,0)$. The runs $t \in[0,22]$ and $t \in[44,66]$ are direct simulations. The run $t \in[22,44]$ is a quotient system simulation, the pinning points are indicated by small white crosses. The third pinning condition is engaged at $t \approx 27.5$.

where only the two translation pinning conditions are engaged, so that the position of the tip of the spiral is fixed, but not its orientation, so the FoR is cotranslating but not corotating. The second part is where all four pinning conditions are engaged, and the FoR is cotranslating and corotating. It is seen from Fig. 3, that after a transient period, the solution in the fully comoving FoR becomes stationary. This corresponds to the definition of a rigidly rotating spiral wave as a relative equilibrium.

Figure 4 shows a similar set of runs for a different case, where the spiral wave is not stationary but is meandering. In this case, the solution in the comoving FoR is not stationary, but periodic in time. This corresponds to the definition of a meandering spiral wave as a relative periodic orbit.

Figures 5(a) and 5(b) show selected pieces of tip trajectories obtained as a result of the runs shown in Figs. 3 and 4. The discretization steps there are deliberately chosen crude to allow very fast running simulations and also to illustrate the difference introduced by the change in method of computation. The tip trajectories obtained by reconstruction from the quotient data are qualitatively similar to the tip trajectories obtained in DNS. However, the quantitative difference is also quite evident. In the case of rigid rotation, the reconstructed trajectory radius is noticeably bigger than that from DNS, and the centers of the meandering patterns in different runs are offset against each other. As panels (c) and (d) in the same figure show, these discrepancies decrease when the discretization steps are refined.

Figure 6 shows the tip and quotient system trajectories, obtained in laboratory and comoving FoR calculations, for a meandering spiral. This is drawn for the finer discretization 


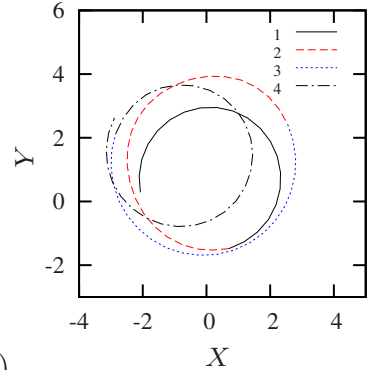

(a)

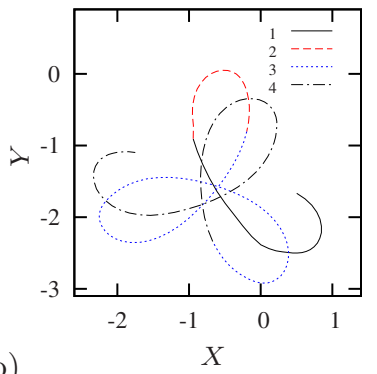

(b)

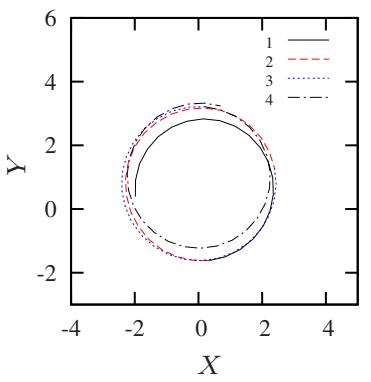

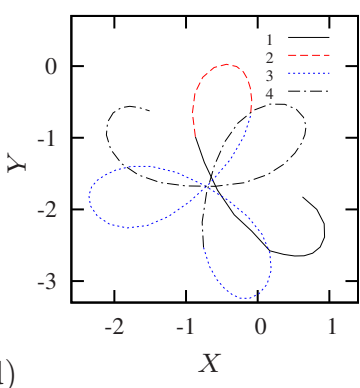

(d)

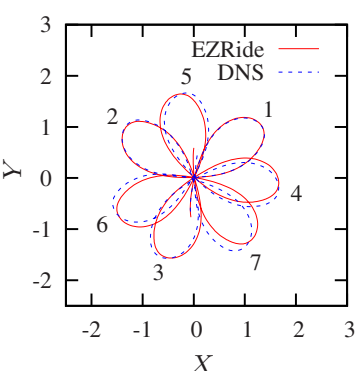

(a)

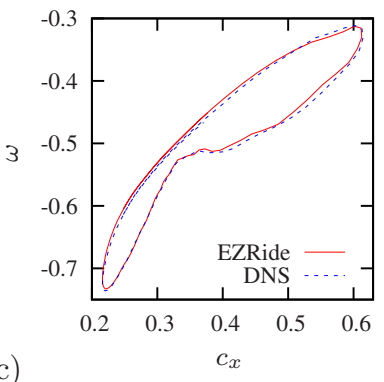

(b)
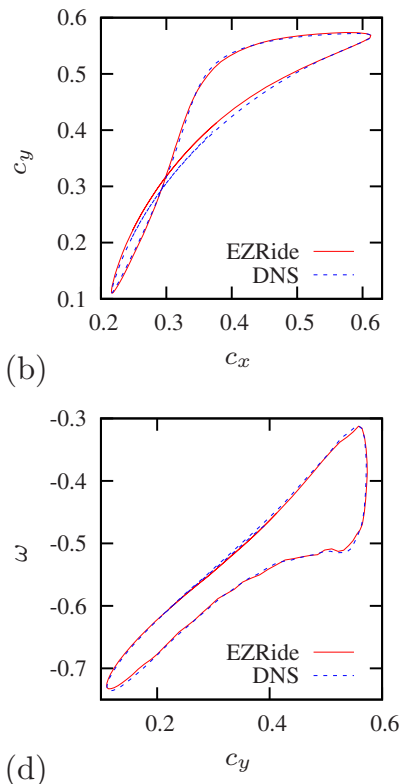

FIG. 6. (Color online) Meander in the FHN model, calculated in the laboratory frame of reference (DNS), and from quotient system (EZRide). In (a), the meandering pattern is shown, which for the EZRide curve is obtained by numerical integration of quotient data using Eq. (15). In (b)-(d), the projections of the quotient data are shown, which for the DNS curves are obtained by numerical differentiation of the tip trajectory, using Eq. (15).

ditions engaged. The pieces labeled 3 correspond to comovin (cotranslating and corotating) simulations with all three pinning conditions engaged. The final pieces labeled 4 correspond to direct simulations in a nonmoving FoR, which has been displaced with respect to the laboratory FoR during the quotient system simulations. (c) Same as (a), with $\Delta_{x}=1 / 10, \Delta_{t}=1 / 4000$. (d) Same as (b), with $\Delta_{x}=1 / 10, \Delta_{t}=1 / 4000$.

steps, as in Fig. 5(d). For comparison, quotient data for both the laboratory and comoving FoR calculations were recalculated for choice 1 pinning conditions using Eq. (29). There is good agreement between the two methods of calculations, within the expected accuracy. More detailed analysis of the numerical accuracy of our method is given in the next session.

\section{NUMERICAL CONVERGENCE}

Figure 7 illustrates the convergence of the results of calculations of rigidly rotating spiral, using EZRide with Neumann and Dirichlet boundary conditions, and DNS using Neumann boundary conditions. In these calculations, the box size is fixed at $L=60$ and the time step is changed with the space step so that $\Delta_{t}=\Delta_{x}^{2} / 40$. For $\omega\left(\Delta_{x}^{2}\right)$ dependence, we also show the angular velocity measured in direct numerical simulations. We do not show $\left|\vec{c}\left(\Delta_{x}^{2}\right)\right|$ found in DNS, since obtaining it involves numerical differentiation which gives accuracy insufficient for the convergence study.

Our discretizations are second order accurate in $\Delta_{x}$ and first order accurate in $\Delta_{t}$ both in DNS and in the riding mode, which corresponds to linear dependence of any results on $\Delta_{x}^{2}$ for $\Delta_{x} \rightarrow 0$. We see in Fig. 7 that this is indeed the case. Linear extrapolation of the $\omega\left(\Delta_{x}^{2}\right)$ gives the values of $\omega(0)$

for laboratory and comoving calculations coinciding to within $10^{-3}$.

One of the advantages of EZRide is the fact that the simulations can be done in a smaller box compared to DNS. So, the last test is convergence in box size. We have calculated the rigidly rotating spiral by EZRide at fixed $\Delta_{x}=1 / 15, \Delta_{t}$ $=1 / 9000$ and $L$ varying through $[15,60]$ and found that both $|\vec{c}|$ and $|\omega|$ vary by less than $10^{-3}$.

\section{APPLICATION I: THE 1:1 RESONANCE IN MEANDERING SPIRAL WAVES}

One of the cases where the DNS would meet with difficulties is the study of the meandering of spiral waves for parameters near the "1:1 resonance" between the Euclidean and the Hopf frequencies. This case is marginal between meandering patterns with inward petals and outward petals.
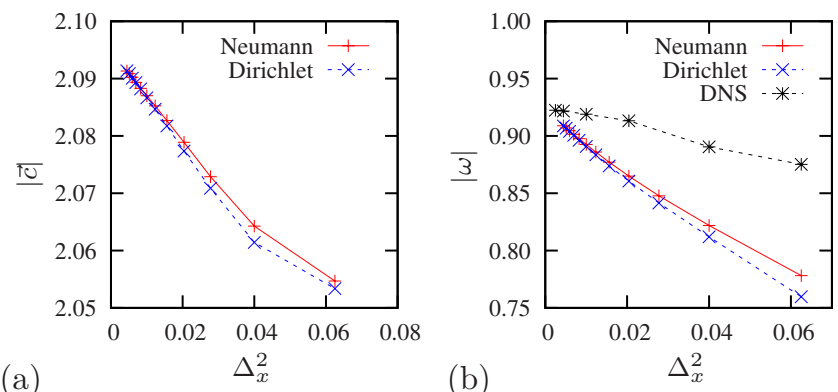

FIG. 7. (Color online) Convergence of the rigidly rotating spiral wave solution in the Barkley's model. 


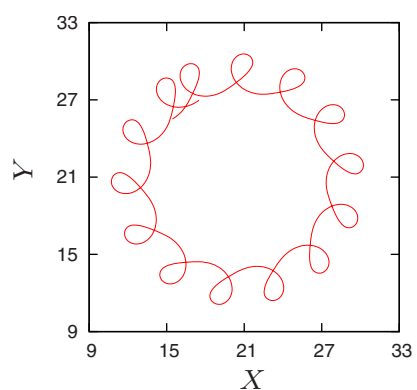

(a) $\quad \beta=0.91535$

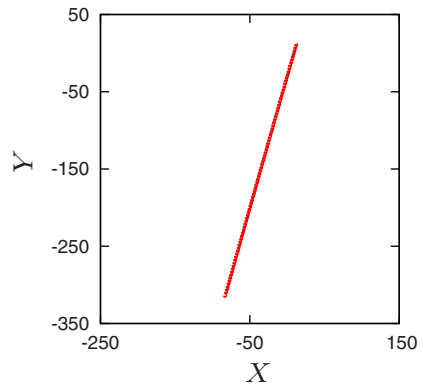

(c) $\quad \beta=0.93535$

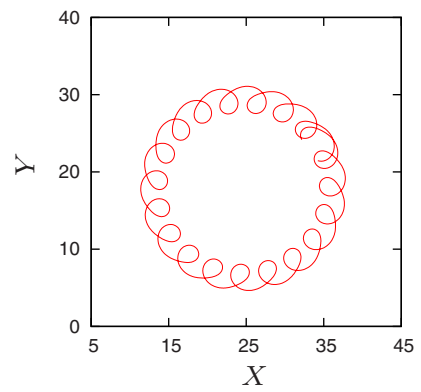

(e)

$$
\beta=0.94535
$$

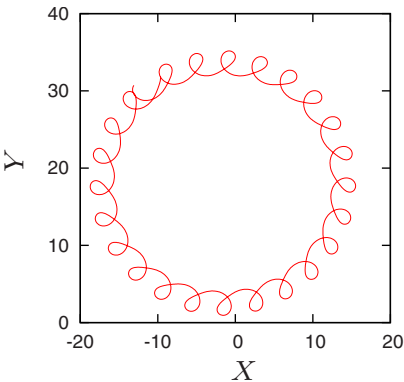

(b) $\quad \beta=0.92535$

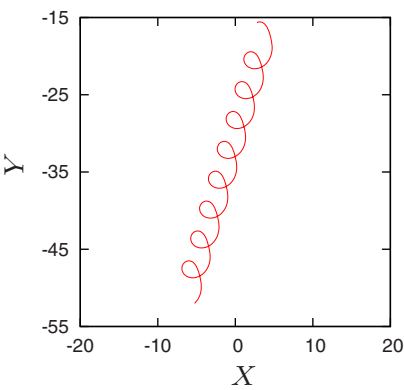

(d)

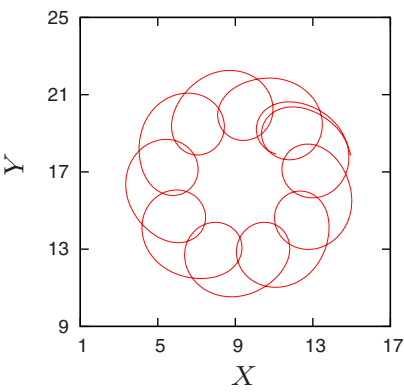

f)

$\beta=0.95535$

FIG. 8. (Color online) The reconstructed tip trajectories in FitzHugh-Nagumo system with $\alpha=0.2, \gamma=0.5$ and varying $\beta$.

Near the resonance, the spatial extent of the meandering trajectory becomes large, and for the case of exact resonance, infinite, and the spiral appears to be spontaneously drifting $[25,43]$. Hence, following the dynamics of the spiral wave in the comoving FoR presents an advantage.

We illustrate this using the FHN model. We fix the discretization parameters at $\Delta_{x}=1 / 8, \Delta_{t}=1 / 2560$, and $L=20$. The choice of model parameter is influenced by Winfree's

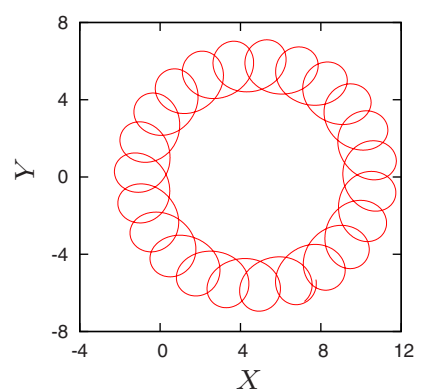

(a) $\beta=0.80362$

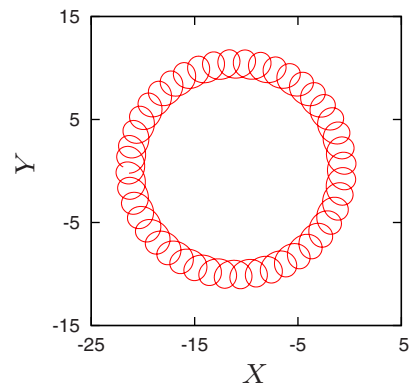

(b) $\quad \beta=0.80862$

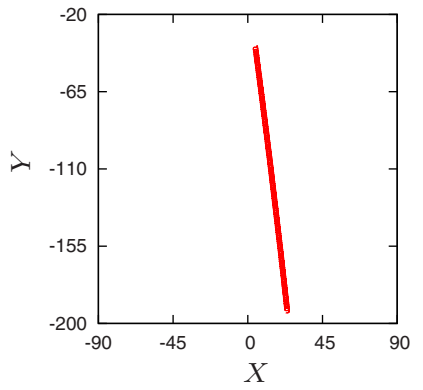

(c)

$$
\beta=0.81362
$$

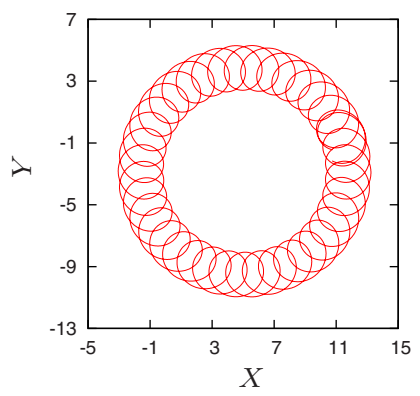

(e) $\quad \beta=0.81862$

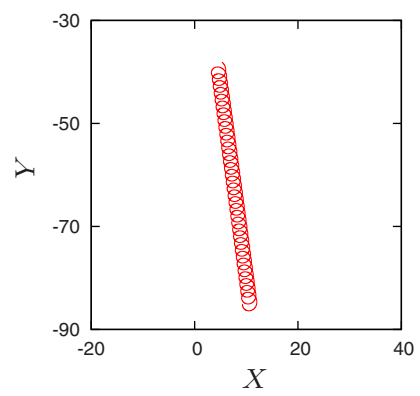

(d)

$\beta=0.81362$

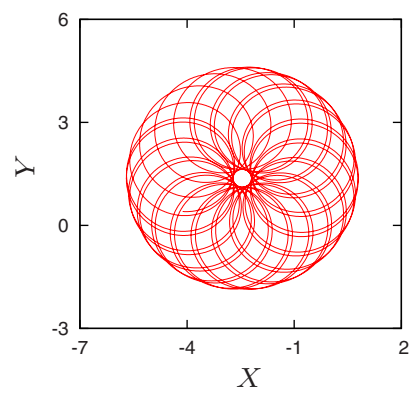

(f)

$\beta=0.82362$

FIG. 10. (Color online) Same as Fig. 8, for $\alpha=0.25$.

"Flower Garden" [44], which gives a rough estimate for the location of the $1: 1$ resonance line in the $(\alpha, \beta)$ plane at $\gamma=0.5$. Using this information, we have selected two values $\alpha=0.2$ and $\alpha=0.25$, and scanned values of $\beta$ across the resonance value, which we determined as $\beta_{0} \approx 0.93535$ for $\alpha=0.2$, and $\beta_{0} \approx 0.81362$ for $\alpha=0.25$ at our discretization parameters.

The results are presented on Figs. 8-11. The shape of trajectories is well known from the theory, and is outward

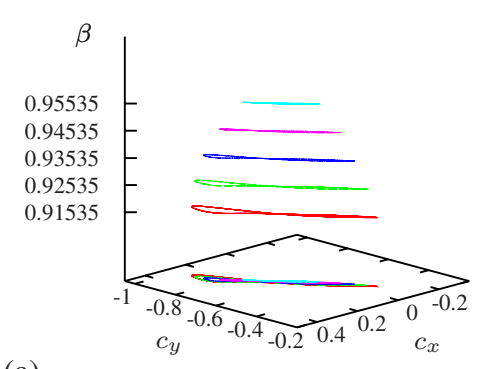

(a)

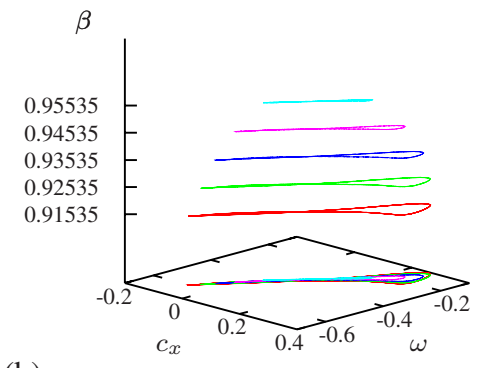

(b)

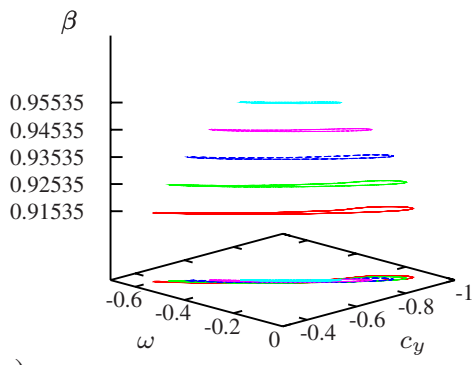

(c)

FIG. 9. (Color online) Various projection of the limit cycles in the quotient system corresponding to the trajectories shown in Fig. 8 . 


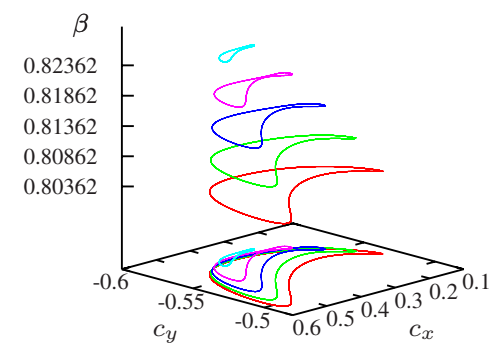

(a)

(b)

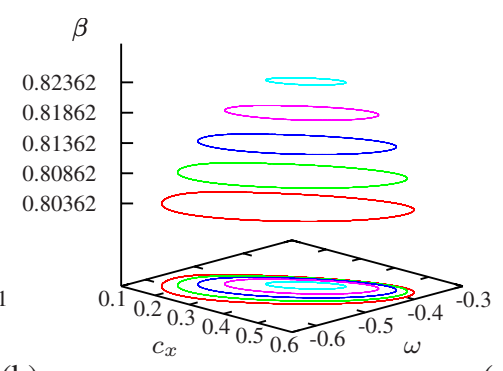

(c)

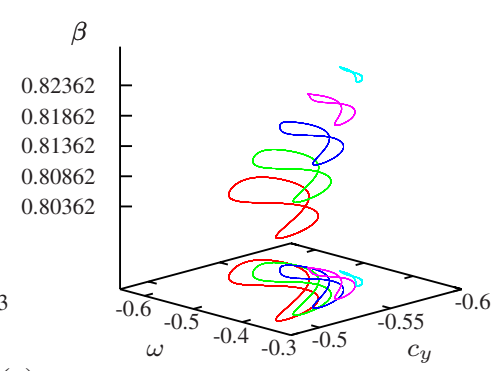

$c_{y}$

FIG. 11. (Color online) Same as Fig. 9, for $\alpha=0.25$.

petals for $\beta<\beta_{0}$ and inward petals for $\beta>\beta_{0}$, degenerating into spontaneous straightforward drift at $\beta=\beta_{0}$. The trajectory at $\beta=\beta_{0}$ in Fig. 8 is shown twice: once for the whole duration as it was calculated, Fig. 8(c), and then a close-up of small part of it, Fig. 8(d). Calculation of this particular trajectory using DNS would require, by our estimate, about five weeks, as opposed to $2.5 \mathrm{~h}$ used by EZRide.

The change in the quotient dynamics with changing $\beta$ is illustrated in Fig. 9. As opposed to the tip trajectories, there is no evident qualitative changes in the shape of the limit cycle across $\beta=\beta_{0}$. Note the very elongated shape of the limit cycles in all three projections. We do not know whether this has some theoretical explanation or is merely incidental.

The parametric line $\alpha=0.25$ exhibits similar behavior, as shown in Figs. 10 and 11. This is closer to the Hopf bifurcation line in the quotient system, called $\partial M$ line in [44]. Correspondingly, the size of the limit cycles in the quotient system is smaller and they become more oval shaped. Note that the scale of $c_{y}$ axis is disproportionately stretched in Fig. 11; i.e., the Hopf central manifold appears to be nearly orthogonal to that axis. Again, there is no qualitative change in the quotient system dynamics when crossing the $1: 1$ resonance.

\section{APPLICATION II: LARGE CORE SPIRALS}

Another example where the spatial extent of the spiral wave dynamics is large is the vicinity of Winfree's "rotors boundary" $\partial R$ in the parametric space [44]. In the vicinity of this boundary, the period of rotation and the radius of the core of the spiral wave grow infinitely.

There are at least two different asymptotic theories, based on different choice of small parameters, which aim to describe the vicinity of $\partial R$. Hakim and Karma [45,46] developed a "free-boundary" asymptotic theory applicable to FitzHugh-Nagumo type models in the limit $c \rightarrow 0$ or $\alpha \rightarrow 0$ in terms of our chosen kinetics, where angular velocity $\omega$ typically decreases as

$$
|\omega| \propto\left|p-p_{*}\right|^{3 / 2}, \quad p \rightarrow p_{*},
$$

where $p$ is a parameter of the model such that $p=p_{*}$ corresponds to the $\partial R$ boundary.

Elkin et al. [47] obtained an alternative asymptotic based on assumptions which were not restricted to kinetics of any particular kind, but which were not directly validated. Their prediction was

$$
|\omega| \propto\left|p-p_{*}\right|, \quad p \rightarrow p_{*} .
$$

Further analysis has suggested that these two alternatives are not actually antagonistic and may be even observed in the same system in different parametric regions [48]. Reliably distinguishing between the two asymptotics is challenging for DNS as it requires a rather close approach to the critical point $p=p_{*}$, which is not known a priori, implying large tip trajectory radii and correspondingly significant computational resources.

In here we present an example of studying this dependence using calculations in the comoving FoR, which is free from the above complication, as it can be performed within the box of fixed size for all $p$.

For this study, we use Barkley's model with varying parameter $p$ chosen to be $a$, varying from $a=0.48$ downwards with step 0.001 until 0.43 , with other parameters fixed at $b$ $=0.05$ and $c=0.02$. The discretization parameters are $L=30$, $\Delta_{x}=1 / 8, \Delta_{t}=1 / 2560$, and $\vec{r}_{\text {inc }}=(0,7 / 4)$.

Selected stationary solution obtained in this way are illustrated in Fig. 12, and the graphs of $\omega(a)$ and $c_{y}(a)$ are shown in Fig. 13. We compare the features of the observed solutions with those that are given by the two asymptotic theories [48], and observe that:

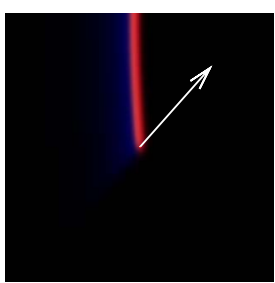

(a) $\quad a=0.430$

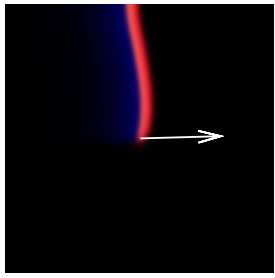

(d) $\quad a=0.456$

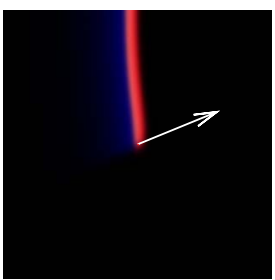

(b) $\quad a=0.450$

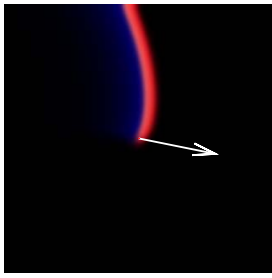

(e) $\quad a=0.460$

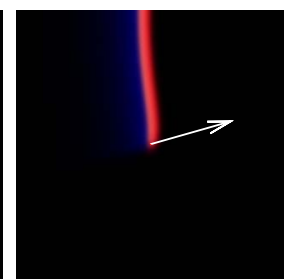

(c) $\quad a=0.453$

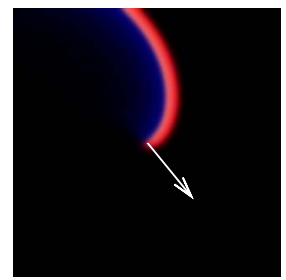

(f) $\quad a=0.480$
FIG. 12. (Color online) Snapshots of relative equilibria in Barkley model obtained at different values of parameter $a$. The arrows indicate the direction of the vector $\vec{c}$. 


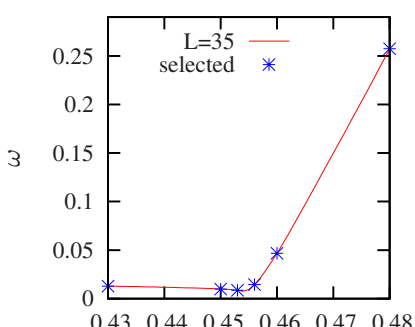

(a)

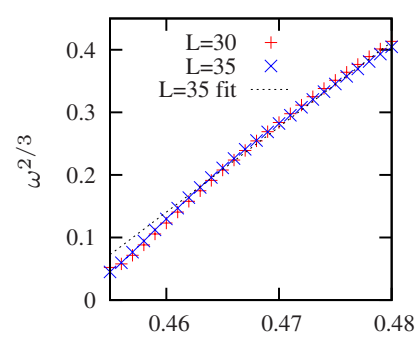

(c)

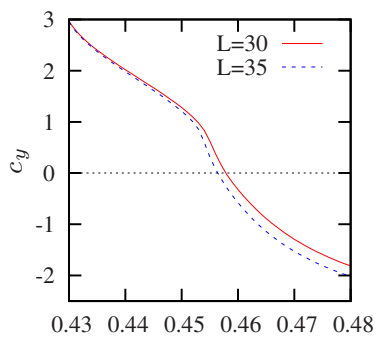

(e)

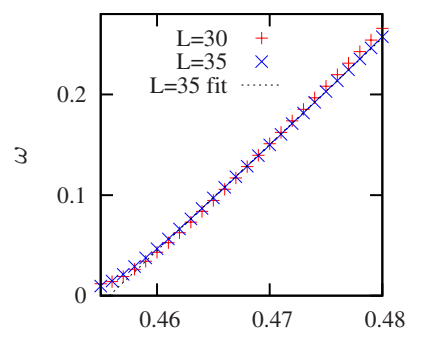

(b)

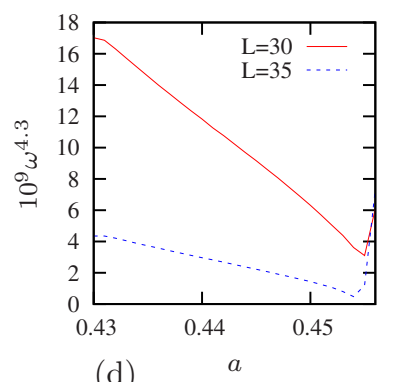

(d)

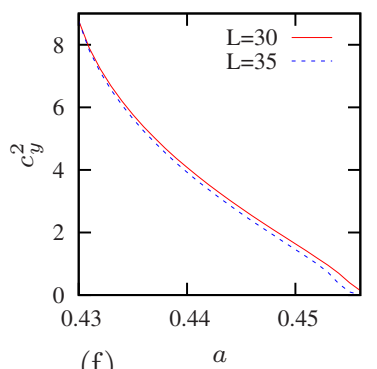

(f)
FIG. 13. (Color online) Dependencies $\omega(a)$ and $c_{y}(a)$ of the relative equilibria, for different $L$ as indicated. On panel (a), the symbols correspond to the selected values of $a$ used in Fig. 12.

(1) there is a critical value of the parameter $a_{*} \approx 0.456$, at which the behavior of the solution changes qualitatively. At $a=a_{*}$, we observe a nearly straight broken excitation wave.

(2) For $a>a_{*}$, the solutions are spiral waves, that is, broken excitation wavelets, which become less and less convex as $a \longrightarrow a_{*}$, and have macroscopic angular velocity which however diminishes in the same limit;

(3) for $a<a_{*}$ the solutions are retracting nearly straight but slightly concave wavelets, with very small angular velocity;

(4) for $a=a_{*}$, the direction of movement of the tip seems approximately orthogonal to the overall orientation of the wave itself;

(5) For $a<a_{*}$, the vertical component of vector $\vec{c}$ depends on $a$ in a way which is consistent with the asymptotic $\left|c_{y}\right|$ $\propto\left|a-a_{*}\right|^{1 / 2}$ [see Figs. 13(e) and 13(f)]. Since the overall orientation of the wavelets, as seen in Figs. 12(a)-12(c), is nearly vertical we can take $c_{y}$ as a crude estimate of the "global tip growth rate" as defined in [48].

(6) For $a>a_{*}$, the angular velocity of solutions depends on $a$ in a way which is consistent with the asymptotic $|\omega| \propto\left|a-a_{*}\right|$, see Fig. 13(b) but not $|\omega| \propto\left|a-a_{*}\right|^{3 / 2}[$ see Fig. 13(c)].

All these observations are in agreement with the theory in [48] and can be used to empirically distinguish between the
Elkin et al. asymptotics (corresponding to the "I/V" parametric boundary in [48]) and Hakim-Karma asymptotics (respectively, "J/C" boundary in [48]).

Feature 1 is inconclusive: existence of a critical solution, called "critical finger" by Hakim and Karma, is common for both $\mathrm{J} / \mathrm{C}$ and $\mathrm{I} / \mathrm{V}$ boundaries, but the shape of this solution is different. It is asymptotically linear for I/V boundary, and asymptotically logarithmic for J/C boundary. Looking at Fig. 12 (d) and considering the effect of the boundary conditions, it is not clear which case is nearer to the observed reality.

Feature 2 is common for $\mathrm{I} / \mathrm{V}$ and $\mathrm{J} / \mathrm{C}$ boundaries. The phenomenological difference is that spirals close to $\mathrm{I} / \mathrm{V}$ boundary can be "growing" or "shrinking," while spirals close to J/C boundary can only be "growing." The movement of the tip in Figs. 12(d)-12(f) seems approximately orthogonal to the orientation of the wavelet near the tip, which is consistent with both cases.

Feature 3 tips the balance in favor the I/V boundary since the broken wavelets are concave. According to [48], the translating waves near an I/V boundary should be concave, and those near an $\mathrm{J} / \mathrm{C}$ boundary should be convex.

Feature 4 is common for $\mathrm{I} / \mathrm{V}$ and $\mathrm{J} / \mathrm{C}$, as in both cases the critical fingers should have zero "global growth rate."

Feature 5 is common for $\mathrm{I} / \mathrm{V}$ and $\mathrm{J} / \mathrm{C}$ boundaries.

Feature 6 is, in our opinion, a convincing evidence in favor of an I/V boundary since according to [48], near I/V boundary the dependence $\omega(\delta)$ is linear, whereas near $\mathrm{J} / \mathrm{C}$ boundary it is $|\omega(\delta)| \propto|\delta|^{3 / 2}$.

An unequivocal interpretation of all theoretical predictions in the view of our present numerical results would require further investigation, as the asymptotics of $[47,48]$ operate with a "crest line" of an excitation wave. There is no obvious operational definition of this line which would be valid up to the tip, and some of the predictions concern the mutual orientation of this line and the tip velocity. However the predictions that can be unambiguously interpreted, seem to indicate that for the model considered here, we have the case of I/V boundary, i.e., Elkin et al. asymptotics, rather than $\mathrm{J} / \mathrm{C}$ boundary corresponding to Hakim-Karma asymptotics.

The last observation here is that of the small angular velocity $\omega$ calculated for the "retracting waves" at $a<a_{*}$ [seen in Figs. 12(a)-12(c)]. As we already noted, the smallness of these $\omega$ values is consistent with the theoretical prediction of translating but not rotating waves. However when these values are magnified, we observe that they demonstrate a peculiar power law $|\omega(a)| \propto\left|a-a_{*}\right|^{p}$ where $p \approx 1 / 4.3$ [see Fig. 13(d)]. A theoretical explanation of this requires further study; it is clear, however, that $\omega$ in this area is strongly affected by the boundaries, as the curves for $L=30$ and $L=35$ differ quite significantly.

\section{APPLICATION III: ELECTROPHORESIS OF MEANDERING SPIRAL}

Finally, we illustrate calculation of the movement of spiral waves in a perturbed reaction-diffusion system. We consider FitzHugh-Nagumo kinetics at the same parameters as in Fig. 4, and add to it "electrophoresis" perturbation (23) in the right-hand side, with $\mathbf{E}=\epsilon \mathbf{D}$. 


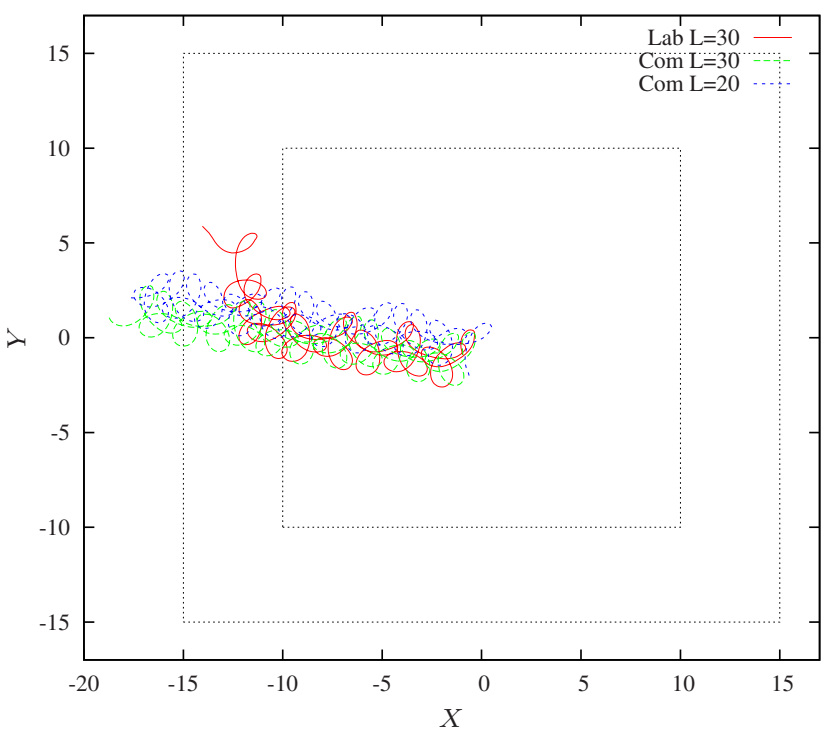

(a)

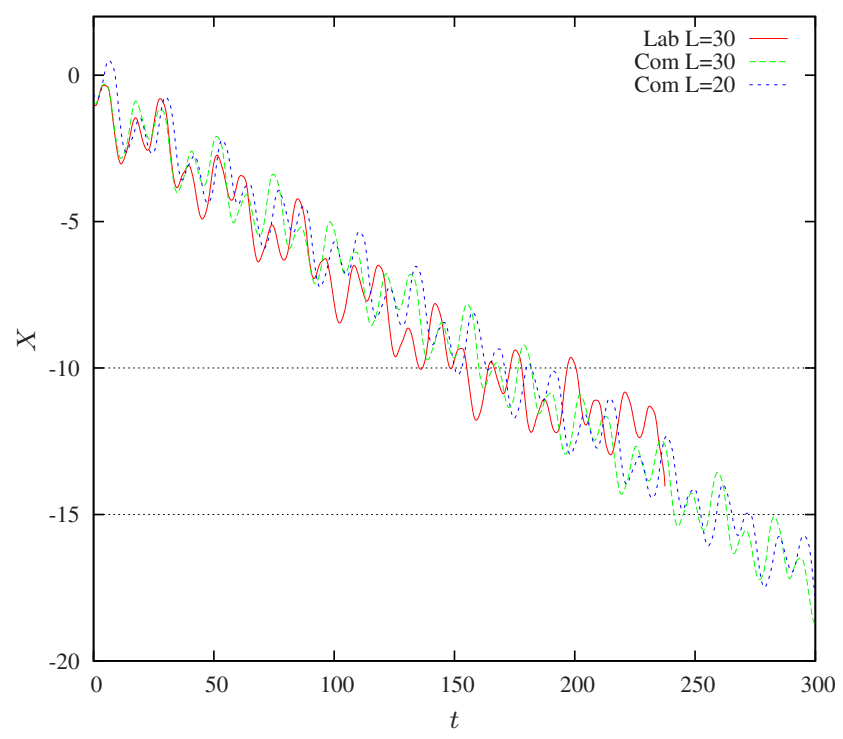

(b)

FIG. 14. (Color online) Trajectories of tips of drifting meandering spirals calculated in the laboratory FoR (for $L=30$ ) and in the comoving FoR (for $L=30$ and $L=20$ ). The thin black dotted lines designate the boundaries of the calculation box in the laboratory FoR where the initial position of the tip is in the center. The parameters are the same as in Fig. 4 and the perturbation is $\mathbf{h}=\epsilon \mathbf{D} \partial_{x} \mathbf{u}$, where $\epsilon=0.1$.

Results of the simulations are presented in Fig. 14. The unperturbed spiral waves for these parameters are meandering, so with the perturbation present, we observe meandering with drift. The drift proceeds with a constant average velocity, which is consistent with the fact that the perturbation violates only the rotational but not the translational symmetry of the problem. The average drift is to the left, which corresponds to a collapsing scroll wave. So at these parameter values, the scroll waves have positive tension, inasmuch as this concept can be applied to meandering scrolls.

In the calculations in the laboratory FoR, the time during which the drift can be observed is limited, as when the spiral reaches the left boundary, it terminates. In the comoving FoR, this drift can be observed indefinitely. Comparing the traces in Fig. 14 we see that although, as we know from Figs. 5 and 6 , the discretization is too crude to give quantitative agreement between laboratory and comoving calculations in detail, the drift velocities obtained in these two ways are very similar.

We illustrate the relative advantages of the two methods of calculation by comparing the computation costs. The laboratory FoR simulation, for $L=30$ and $t \in[0,300]$ has taken $325 \mathrm{~s}$ (the spiral has annihilated at the left at $t \approx 237$ ). The time taken by the comoving FoR simulation for the same box size $L$ and the same $t$ interval is 462 s, i.e., is naturally somewhat longer due to the extra effort required for the advection term calculations. However, the comoving FoR calculation proceeded unabated where the laboratory FoR calculation failed due to annihilation with the border. To continue the laboratory FoR calculation to the same extent we would have to increase the box size $L$ with a corresponding increase in computation cost. Moreover, virtually the same result, as far as drift velocity is concerned, can be obtained by comoving FoR calculation with $L=20$, and it takes only 202 s. Of course the drift in the laboratory FoR with $L=20$ would terminate even earlier.

\section{DISCUSSION}

We have described a numerical method of solving a reaction-diffusion system of equations describing a spiral wave in a frame of reference which is moving with the tip of that wave.

We have shown the method can provide accurate solutions, and that there are applications where the computational cost of our method can be considerably lower than that of the conventional approach, or the conventional approach is just inapplicable. As always, the computational advantages are particularly essential in case of parametric studies, for which the method is well suited. Although the applications were chosen just to provide some meaningful examples of use of the method, the results obtained there can be of scientific value themselves.

So, we have investigated the vicinity of the "1:1 resonance" manifold in the parametric space, which corresponds to spontaneous drift of spirals, and which separates meandering patterns with outward petals and inward petals. Henry [49] proposed a theory which implies that this manifold coincides or is an analytical continuation of the manifold where the filament tension of scroll waves vanishes. There are reports in literature confirming that change of sign of filament tension is associated with change from outward to inward petals in meandering patterns, but also examples where there are no such correlation, e.g., [50] and references therein. Our simulations indicate that as far as orbit manifold dynamics of the spiral is concerned, the 1:1 resonance is not characterized by any special features. Hence any special features of this resonance ought to be due to the Euclidean extension of the orbifold dynamics. Since scroll filament tension can also be defined via properties of the spiral wave solutions within the comoving FoR, any genetic and generic relationship between the two manifolds seems unlikely (but, of course, one cannot exclude the possibility of such relationship in some special cases). 
We have also investigated the vicinity of the " $\partial R$ " manifold in the parametric space, which has provided a strong evidence toward one of the two theoretical possible asymptotics, namely, Elkin et al. [47] asymptotics as opposed to Hakim-Karma [45] asymptotics. It should be noted here that while Hakim-Karma asymptotic theory was based on assumptions which have been well established, the Elkin et al. asymptotic theory was using assumptions, validity of which could not be asserted at that moment. Here we have presented firm evidence that Elkin et al. asymptotics is not a mere theoretical possibility but is indeed observed in reality (see also [36] and a discussion below). A direct confirmation would be via calculation of the "response functions," i.e., critical eigenfunctions of the adjoint linearized operator of the critical finger solution. This would require obtaining first a good quality critical finger solution, so the method described here can be a significant step toward this goal, too.

Finally, we have demonstrated that calculations in the comoving FoR can be efficiently used to study perturbationcaused drift of spirals, including meandering spirals. Although the asymptotic theory of drift of meandering spirals is yet to be developed (see, however, a preliminary draft of such theory in [51]), we can expect, for instance, that scroll waves in the FitzHugh-Nagumo model with the parameters as in Figs. 4 and 14 will have "positive tension," i.e., tend to collapse, rather than develop a scroll wave turbulence. The advantage of calculating drift in the comoving FoR, apart from computation cost, is absence of "pinning" effects of spatial discretization, both in terms of discrete space steps and discrete spatial directions, on the drift.

Our approach can be compared to the approach proposed by Beyn and Thummler (BT) [35]. BT use a similar mathematical idea of decomposing the evolution of the nonlinear wave into the motion of the wave and evolution of its shape, which in the functional space appears as decomposition into motion along and across the Euclidean symmetry group orbits. But there are also differences. There are technical details of implementations which are probably of lesser importance, such as choice of polar vs Cartesian grid, central vs upwind discretization of spatial derivatives and explicit vs semi-implicit discretization in time. More significant differences are in the "phase conditions" they use, which play the same role as, but are qualitatively different in nature from, our "pinning conditions." One aspect is that the phase conditions involve integral functionals. We show here that this is not necessary, and local conditions such as Eq. (24) are simpler. The other aspect is the one we discussed in Sec. II: the BT phase conditions appear to be well suited for calculation of relative equilibria (rigidly rotating spirals) but not necessarily for relative periodic solutions (meandering spirals). Further, the phase conditions proposed by BT were not intended for use with symmetry-breaking perturbations that produce drift of spirals. And indeed, BT comment in their paper that "it seems quite a challenging task to freeze drifting spirals or recognize meandering spirals as periodic orbits." As we have demonstrated, our approach works both for meandering spirals and for drifting spirals.

After completing this study we became aware of a work by Hermann and Gottwald (HG) [36] who also investigated the large core limit, using a further development of the BT method. HG paid a great deal of attention on refining the boundary conditions so as to minimize the effect of boundaries onto the quotient dynamics. This has allowed them, in particular, to verify linear scaling law (32) for seven decades of variation in $|\omega|$, compared to mere one decade as shown in Fig. 13. Notice that as shown in the same figure, our progress toward smaller values of $|\omega|$ is limited precisely by the influence of boundaries. HG also have explicitly addressed the issue of the numerical stability of the computations, which we treat in this study purely empirically.

We believe that combining the advantageous features of the approach developed by BT and HG, and the one proposed here, is an interesting topic for future work, which may yield further results about spiral wave dynamics, that are not possible, or very difficult, to obtain by direct numerical simulations.

\section{ACKNOWLEDGMENTS}

This study has been supported in part by EPSRC-GB Grant No. EP/D074789/1. The preliminary stage for this study was done in collaboration with B. N. Vasiev, who proposed the idea of solving for quotient data simultaneously with the advection substep. V.N.B. is also grateful to A. M. Pertsov and G. A. Gottwald for inspiring discussions, and to G. A. Gottwald also for informing us about the results of [36] prior to publication.
[1] A. M. Zhabotinsky and A. N. Zaikin, in Oscillatory Processes in Biological and Chemical Systems, edited by E. E. Selkov, A. A. Zhabotinsky, and S. E. Shnol (Nauka, Pushchino, 1971), p. 279, in Russian.

[2] M. A. Allessie, F. I. M. Bonke, and F. J. G. Schopman, Circ. Res. 33, 54 (1973).

[3] F. Alcantara and M. Monk, J. Gen. Microbiol. 85, 321 (1974).

[4] A. B. Carey, R. H. Giles, Jr., and R. G. McLean, Am. J. Trop. Med. Hyg. 27, 573 (1978).

[5] N. A. Gorelova and J. Bures, J. Neurobiol. 14, 353 (1983).

[6] L. S. Schulman and P. E. Seiden, Science 233, 425 (1986).
[7] J. D. Murray, E. A. Stanley, and D. L. Brown, Proc. R. Soc. London, Ser. B 229, 111 (1986).

[8] B. F. Madore and W. L. Freedman, Am. Sci. 75, 252 (1987).

[9] S. Jakubith, H. H. Rotermund, W. Engel, A. von Oertzen, and G. Ertl, Phys. Rev. Lett. 65, 3013 (1990).

[10] J. Lechleiter, S. Girard, E. Peralta, and D. Clapham, Science 252, 123 (1991).

[11] T. Frisch, S. Rica, P. Coullet, and J. M. Gilli, Phys. Rev. Lett. 72, 1471 (1994)

[12] A. G. Shagalov, Phys. Lett. A 235, 643 (1997).

[13] D. J. Yu, W. P. Lu, and R. G. Harrison, J. Opt. B: Quantum 
Semiclassical Opt. 1, 25 (1999).

[14] K. Agladze and O. Steinbock, J. Phys. Chem. A 104, 9816 (2000).

[15] M. A. Dahlem and S. C. Müller, Biol. Cybern. 88, 419 (2003).

[16] O. A. Igoshin, R. Welch, D. Kaiser, and G. Oster, Proc. Natl. Acad. Sci. U.S.A. 101, 4256 (2004).

[17] Y. Larionova, O. Egorov, E. Cabrera-Granado, and A. EstebanMartin, Phys. Rev. A 72, 033825 (2005).

[18] P. Oswald and A. Dequidt, Phys. Rev. E 77, 051706 (2008).

[19] T. Bretschneider, K. Anderson, M. Ecke, A. MüllerTaubenberger, B. Schroth-Diez, H. C. Ishikawa-Ankerhold, and G. Gerisch, Biophys. J. 96, 2888 (2009).

[20] V. N. Biktashev, Ph.D. thesis, Moscow Institute of Physics and Technology, 1989 (http://www.maths.liv.ac.uk/ vadim/theses/Biktashev.pdf).

[21] V. N. Biktashev, A. V. Holden, and H. Zhang, Philos. Trans. R. Soc. London, A 347, 611 (1994).

[22] V. Biktashev and A. Holden, Chaos, Solitons Fractals 5, 575 (1995).

[23] I. V. Biktasheva, Y. E. Elkin, and V. N. Biktashev, Phys. Rev. E 57, 2656 (1998).

[24] I. V. Biktasheva and V. N. Biktashev, Phys. Rev. E 67, 026221 (2003).

[25] D. Barkley, Phys. Rev. Lett. 72, 164 (1994).

[26] D. Barkley and I. G. Kevrekidis, Chaos 4, 453 (1994).

[27] D. Barkley, in Chemical Waves and Patterns, edited by R. Kapral and K. Showalter (Kluwer, New York, 1995), pp. 163187.

[28] R. M. Mantel and D. Barkley, Phys. Rev. E 54, 4791 (1996).

[29] V. N. Biktashev, A. V. Holden, and E. V. Nikolaev, Int. J. Bifurcation Chaos Appl. Sci. Eng. 6, 2433 (1996).

[30] C. Wulff, Ph.D. thesis, Freie Universität Berlin, 1996.

[31] P. Chossat, Acta Appl. Math. 70, 71 (2002).

[32] D. Barkley, Physica D 49, 61 (1991).
[33] D. Barkley, EZ-SPIRAL: A Code for Simulating Spiral Waves, http://www.warwick.ac.uk/ masax/Software/ez_software.html

[34] A. J. Foulkes and V. N. Biktashev, EZRide: A Code for Simulating Spiral Waves in Comoving Frame of Reference, http:// www.maths.liv.ac.uk/ vadim/software/EZRide/

[35] W. J. Beyn and V. Thummler, SIAM J. Appl. Dyn. Syst. 3, 85 (2004).

[36] S. Hermann and G. A. Gottwald (private communication); e-print arXiv:1003.5830.

[37] V. N. Biktashev and A. V. Holden, Physica D 116, 342 (1998).

[38] M. Nicol, I. Melbourne, and P. Ashwin, Nonlinearity 14, 275 (2001).

[39] P. Ashwin, I. Melbourne, and M. Nicol, Physica D 156, 364 (2001).

[40] R. FitzHugh, Biophys. J. 1, 445 (1961).

[41] J. Nagumo, S. Arimoto, and S. Yoshizawa, Proc. IRE 50, 2061 (1962).

[42] D. Barkley, M. Kness, and L. S. Tuckerman, Phys. Rev. A 42, 2489 (1990).

[43] V. S. Zykov, Biophysics (Engl. Transl.) 31, 940 (1986).

[44] A. T. Winfree, Chaos 1, 303 (1991).

[45] V. Hakim and A. Karma, Phys. Rev. Lett. 79, 665 (1997).

[46] V. Hakim and A. Karma, Phys. Rev. E 60, 5073 (1999).

[47] Y. E. Elkin, V. N. Biktashev, and A. V. Holden, Chaos, Solitons Fractals 9, 1597 (1998).

[48] Y. E. Elkin, V. N. Biktashev, and A. V. Holden, Chaos, Solitons Fractals 14, 385 (2002).

[49] H. Henry, Phys. Rev. E 70, 026204 (2004).

[50] S. Alonso and A. V. Panfilov, Phys. Rev. Lett. 100, 218101 (2008).

[51] A. Foulkes, Ph.D. thesis, University of Liverpool, 2009; e-print arXiv:0912.4247.

[52] We are thankful to B.N. Vasiev for this idea. 